\title{
Dark solitons in nonlinear media with arbitrary degree of nonlocality
}

\author{
Qian Kong ${ }^{1,2}$, Qi Wang ${ }^{2}$, Ole Bang ${ }^{3}$, Wieslaw Krolikowski ${ }^{1}$ \\ 1. Laser Physics Center, Research School of Physics and Engineering, Australian National University, Canberra ACT 0200, Australia \\ 2. Department of Physics, Shanghai University, Shanghai 200444, China \\ 3. DTU Fotonik, Department of Photonics Engineering, Technical University of Denmark, 2800 Kgs. Lyngby, Denmark
}

Spatial dark optical solitons are localized light intensity dips in the infinite constant background [1]. They exist as a result of a balance between diffraction and nonlinearity of the medium. In recent years there has been strong interest in the so-called nonlocal nonlinearities, in which the nonlinearity in a particular spatial location is determined by the light intensity in a certain neighborhood of this location.

Here we describe analytically, for the first time to our knowledge, properties of dark nonlocal solitons. To this end we employ a variational approach and show that it enables one to retrieve the major features of dark solitons in a general nonlocal regime. And we also investigate analytically the interaction of dark solitons in nonlocal media with an arbitrary degree of nonlocality. Our results clearly show how nonlocality induces an attractive force, which depends on the degree of nonlocality and counteracts the otherwise inherent repulsive nature of dark soliton interaction.

The evolution of a one-dimensional optical beam with an amplitude $u(x, z)$ in a nonlocal defocusing medium is governed by the following nonlocal nonlinear Schrödinger equation

$$
i \frac{\partial u}{\partial z}+\frac{1}{2} \frac{\partial^{2} u}{\partial x^{2}}-u \int_{-\infty}^{+\infty} R(x-\xi)|u(\xi, z)|^{2} d \xi=0,
$$

where $\mathrm{R}(\mathrm{x})$ is the nonlocal response function. In order to make the problem analytically tractable we will consider here the particular model of nonlocality described by the rectangular nonlocal response function

$$
R(x)= \begin{cases}\frac{1}{2 \sigma} & -\sigma \leq x \leq+\sigma, \\ 0 & \text { otherwise }\end{cases}
$$

In order to describe analytically the properties of solitons we use the variational approach. From the Lagrangian representation of the Eq.(1) we derive ordinary differential equations governing the evolution of soliton parameters such as velocity, width and position. Moreover, they also allow one to investigate the interaction of dark nonlocal solitons. It is well known that dark solitons in local media always repel. However, here we are able to show theoretically that nonlocality provides the attractive force between solitons and may even lead to the formation of the soliton bound state. In the graph (a) we plot the effective potential governing the soliton interaction for two values of the degree of nonlocality $\sigma$. It is clear that while this potential is repulsive for close soliton separation it exhibits attraction for larger separation. The minimum of the effective potential corresponds to the soliton bound state. In the graph (b) we show the analytically obtained bound-state separation between two dark solitons as a function of degree of nonlocality. These analytical predictions were subsequently verified numerically using the splitstep Fourier method to integrate the nonlinear equation Eq.(1) with the initial conditions representing two non-interacting dark solitons. Exemplary results are shown in the graphs (c-e)
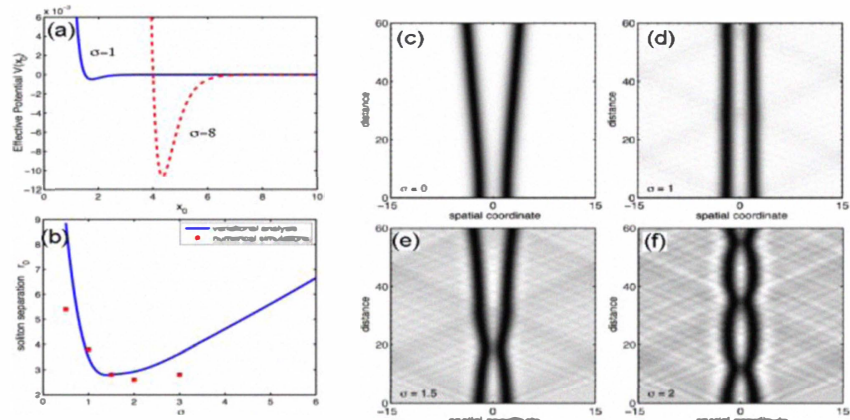

which depict propagation of both solitons as a function of the degree of nonlocality $\sigma=0,1,1.5$ and 2 , respectively. It is clear that as the extent of nonlocal response increases both solitons start experiencing the attractive force. We found numerically soliton separation corresponding to their bound state and compared it with the analytical results of figure (b). Numerical solutions are indicated by squares. It is evident that both analytical approach and numerics qualitatively agree.

\section{References}

[1] Y. S. Kivshar and B. Luther-Davies, "Dark optical solitons: physics and applications", Phys. Rep. 298, 81 (1998) and references therein. [2] Q. Kong, Q. Wang, O. Bang, and W. Krolikowski, "Analytical theory of dark nonlocal solitons", Opt. Lett. 35, 2152 (2010).

[3] Q. Kong, Q. Wang, O. Bang, and W. Krolikowski, "Analytical theory for the dark-soliton interaction in nonlocal nonlinear materials with an arbitrary degree of nonlocality", Phys. Rev. A 82, 013826 (2010). 